\title{
Effects of Double-Chained Cationic Surfactants n-Dimethyldialkylammoniums on Skin Permeation of Benzoic Acid through Excised Guinea Pig Dorsal Skin: Comparison of Their Enhancement Effects with Hemolytic Effects on Erythrocytes
}

\author{
Shuji Kitagawa, * Masatoshi Kasamaki, and Fuyuko HiYama \\ Niigata College of Pharmacy, 5-13-2 Kamishin'ei-cho, Niigata 950-2081, Japan. \\ Received April 16, 2001; accepted June 9, 2001
}

We examined the effects of the double-chained cationic surfactants dimethyldialkylammoniums $\left(\mathrm{CH}_{3}\right)_{2} \mathrm{~N}^{+}\left(\mathrm{C}_{n} \mathrm{H}_{2 n+1}\right)_{2}$ on the permeation of benzoic acid through excised guinea pig dorsal skin. Among five dimethyldialkylammoniums tested $(n=10-18)$, dimethyldidecylammonium $(n=10)$ had dose-dependent enhancement effects at concentrations of more than $20 \mu \mathrm{M}$. Compared with the marked enhancement effects of dimethyldialkylammoniums with relatively shorter alkyl chains, those of long-chain dimethyldialkylammoniums $(n=16$, 18) were much less. We compared the enhancement effects of these cationic surfactants on skin permeation with their hemolytic effects on guinea pig erythrocytes. Their enhancement effects corresponded to their hemolytic activity. The findings suggest that dimethyldialkylammoniums with relatively shorter alkyl chains, which form either vesicles with looser molecular packing or micelles and appear to be present as surfactant monomers in higher ratios than those with longer alkyl chains, favor the interaction with skin. Their enhancement mechanism is possibly similar to that of single-chained cationic surfactants.

Key words dimethyldialkylammonium; cationic vesicle; skin absorption; benzoic acid; hemolysis; ESR

It has been reported that cationic surfactants such as dodecyltrimethylammonium have marked enhancement effects on transdermal drug permeation compared with anionic surfactants such as sodium dodecylsulfate. ${ }^{1,2)}$ Among cationic surfactants, the effects of double-chained cationic surfactants such as dimethyldialkylammoniums, which have been used as antistatic agents, ${ }^{3)}$ are still unknown. Various types of double-chained cationic surfactants have been shown to form vesicles and used for gene delivery. ${ }^{4,5)}$ Therefore their effects on skin absorption may differ from those of single-chained cationic surfactants, such as cetyltrimethylammonium, which form micelles above critical micelle concentrations. Since skin has high ratio of negatively charged lipid components in the stratum corneum lipid lamella which works as a barrier to drug absorption, ${ }^{6,7)}$ they may interact with these components in the stratum corneum and modify drug permeation either as vesicles or as surfactant monomers.

In this study we examined the effects of five dimethyldialkylammoniums $\left(\mathrm{CH}_{3}\right)_{2} \mathrm{~N}^{+}\left(\mathrm{C}_{n} \mathrm{H}_{2 n+1}\right)_{2}$, which have different lengths of alkyl chains $(n=10-18)$, on in vitro skin permeation using benzoic acid as a model drug. Since erythrocytes have been used as a model system to study the interaction of surfactants with the lipid lamella of the stratum corneum, ${ }^{8)}$ we also examined their hemolytic effects on erythrocytes to reveal the mechanism of their effects on skin permeation. We further examined the molecular assemblies of dimethyldialkylammoniums by observing ESR spectra using 5-doxylstearic acid (5-NS) as a spin label.

\footnotetext{
Experimental

Materials Bromide salts of dimethyldialkylammoniums were purchased from Tokyo Chemical Industry Co., Ltd. (Tokyo, Japan). 5-NS was purchased from Aldrich (Milwaukee, WI, U.S.A.). Benzoic acid and all other reagents were purchased from Wako Pure Chemical Industries (Osaka, Japan)
}

Preparation of Sonicated Surfactants Bromide salts of dimethyldialkylammoniums were dissolved in chloroform, and the solvent was evaporated. Dried surfactant films were prepared by removing the solvent under vacuum evaporation. The cationic surfactants were hydrated and suspended by voltex mixing in phosphate-buffered saline (PBS). Then the suspension was sonicated with a probe-type sonicator for $5 \mathrm{~min}$ at an output power of $80 \mathrm{~W}$ at $50^{\circ} \mathrm{C}$.

Measurement of in Vitro Skin Permeation In vitro skin permeation of drugs was examined as described previously. ${ }^{9)}$ Full-thickness dorsal skin was excised from male guinea pigs and subcutaneous fat and other extraneous tissues were trimmed. The skin was then mounted in a two-chamber diffusion cell with a water jacket $\left(37^{\circ} \mathrm{C}\right)$. The available mean diffusion area was $0.65 \mathrm{~cm}^{2}$, and mean half-cell volume was $5.4 \mathrm{ml}$. The donor cells were filled with saline either in the presence or absence of sonicated surfactants and the receiver cells filled with PBS ( $\mathrm{pH} 7.4)$. Both donor and receiver cells were stirred at $450 \mathrm{rpm}$ with a magnetic stirrer during 12-h pretreatment of the skin. After washing both donor and receiver cells, a suspension of excess amounts of benzoic acid in saline either in the presence or absence of the sonicated surfactants was added to the donor compartments, and the permeation experiment was started. The $\mathrm{pH}$ value in the donor suspension was 2.6, which was significantly lower than the $\mathrm{p} K_{\mathrm{a}}$ value of benzoic acid (4.2). ${ }^{10}$ ) One hundred fifty microliters of sample was taken from the receiver cells periodically over a maximum period of $9 \mathrm{~h}$, then diluted 20 times or more with PBS and analyzed by UV absorbance at $224 \mathrm{~nm}$. Solubilized components from skin did not interfere with the UV absorbance.

Steady-state flux $J$ of benzoic acid was obtained according to Eq. 1 from the initial straight portion of the permeation curve $\mathrm{d} C_{\mathrm{R}} / \mathrm{d} t$.

$$
J=\frac{\mathrm{d} C_{\mathrm{R}}}{\mathrm{d} t} \cdot \frac{V_{\mathrm{R}}}{A}
$$

where $C_{\mathrm{R}}$ and $V_{\mathrm{R}}$ are the concentration of benzoic acid in the receiver compartment and the compartment volume, respectively, and $A$ is the diffusion area. The apparent permeability coefficient $K_{\mathrm{p}}$ was calculated by the results of Eq. 1 and the solubility $C_{\mathrm{d}}$, which was measured as described previously, ${ }^{11)}$ from the following equation.

$$
K_{\mathrm{p}}=J / C_{\mathrm{d}}(2)
$$

Measurement of ESR Spectra Measurement of ESR spectra was carried out using 5-NS as a spin label. The sonicated surfactant prepared in PBS was incubated with 5 -NS $25 \mu \mathrm{M}$ at $37^{\circ} \mathrm{C}$ for $2 \mathrm{~min}$ and transferred to duplicate 20- $\mu 1$ capillaries. One end of the capillaries was sealed with Hematoseal (Terumo, Tokyo, Japan) and inserted into the ESR tubes. ESR spectra were measured at $37^{\circ} \mathrm{C}$ with a TE-200 (X-band) spectrometer (JEOL, Tokyo, Japan) with 100-kHz field modulation frequency and 0.1-mT modulation amplitude at an output power of $8 \mathrm{~mW}$ as reported previously. ${ }^{12}$ The order parameter of 5-NS was calculated from ESR spectra as 
described. ${ }^{12}$

Measurement of Hemolysis of Erythrocytes Fresh guinea pig blood was drawn into acid citrate-dextrose (ACD) anticoagulant solution $(2.2 \%$ sodium citrate, $0.8 \%$ citric acid, and $2.2 \%$ dextrose) and centrifuged at $2000 \times \boldsymbol{g}$ for $5 \mathrm{~min}$ at $4{ }^{\circ} \mathrm{C}$ to remove plasma and the buffy coat. The erythrocytes in the pellet were then washed three times with PBS by centrifugation at $2000 \times \boldsymbol{g}$ for $5 \mathrm{~min}$ and collected by centrifugation at $4500 \times \boldsymbol{g}$ for $8 \mathrm{~min}$. The erythrocytes were suspended in PBS at a hematocrit value of about $20 \%$ and incubated in the presence of the sonicated surfactants at $37^{\circ} \mathrm{C}$. An aliquot of this mixture was removed after 1-min or 60 -min incubation. The extent of hemolysis was measured by hemoglobin release in the supernatant after centrifugation at $2000 \times \boldsymbol{g}$ for $2 \mathrm{~min}$. Hemoglobin was determined spectrophotometrically at $541 \mathrm{~nm}$ according to the cyanmethemoglobin method. ${ }^{13)}$ Aggregation of the erythrocytes was observed by Olympus phasecontrast microscopy equipment (Tokyo, Japan).

\section{Results}

Effects of Dimethyldialkylammoniums on Skin Absorption of Benzoic Acid We examined the effects of different concentrations of dimethyldialkylammoniums $\left(\mathrm{CH}_{3}\right)_{2} \mathrm{~N}^{+}-$ $\left(\mathrm{C}_{n} \mathrm{H}_{2 n+1}\right)_{2}$ on the flux of benzoic acid, which was mostly present as an undissociated form under these experimental conditions. As shown in Fig. 1, dimethyldidecylammonium (DMDDA) dose dependently increased the flux in the concentration range of more than $20 \mu \mathrm{M}$ and increased the flux more than three-fold at $5 \mathrm{~mm}$. The apparent permeability coefficient also increased to a similar extent in the same DMDDA concentration range, as shown in Fig. 2. A slight

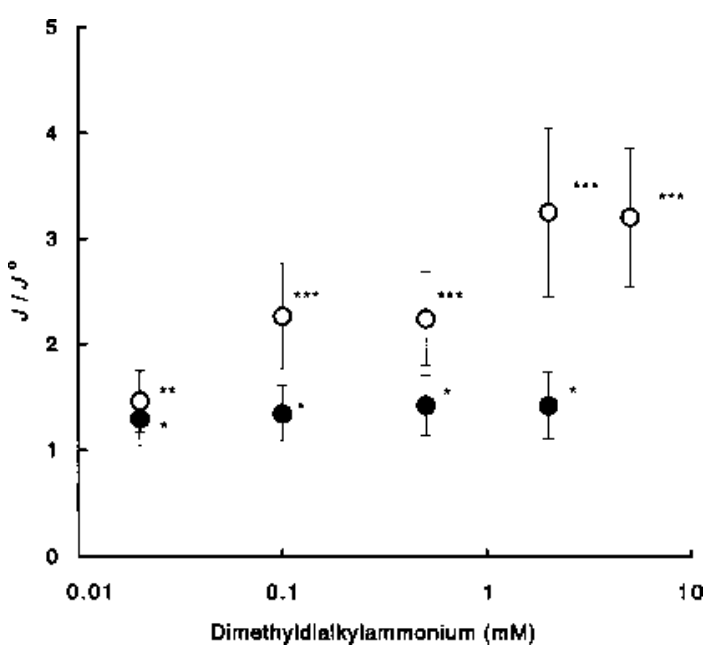

Fig. 1. Dose-Dependent Effects of DMDDA (O) and DMDPA ( $(\bullet)$ on the Flux $(J)$ of Benzoic Acid

Data, which represent the ratio to the flux in the absence of dimethyldialkylammoniums $\left(J^{0}\right)$, are means \pm S.D. of four experiments. The significance of the difference between the flux of benzoic acid without the surfactant and that with the surfactant was determined by Student's $t$-test. $* p<0.05, * * p<0.01, * * * p<0.001$. increase in the solubility of benzoic acid was observed in the same concentration range as shown for its effect at $2 \mathrm{~mm}$ in Table 1.

On the other hand, dimethyldipalmitylammonium (DMDPA), which has longer alkyl chains $(n=16)$, only slightly increased the flux, as shown in Fig. 1. Similarly, only a slight tendency of increase in the apparent permeability coefficient was observed, as shown in Fig. 2. The solubility of benzoic acid increased slightly in the presence of DMDPA.

As shown in Table 1 for the effects at $2 \mathrm{~mm}$, dimethyldilaurylammonium (DMDLA) and dimethyldimyristylammonium (DMDMA), which have relatively shorter alkyl chains, significantly increased the flux and permeability coefficient of benzoic acid, as observed with DMDDA. The enhancement effects of these three double-chained cationic surfactants at $2 \mathrm{~mm}$ were similar to those of the single-chained cationic surfactants like cetyltrimethylammonium (CTMA), ${ }^{2)}$ the effects of which are also shown in Table 1 for comparison. On the other hand, the enhancement effects of dimethyldialkylammoniums with longer alkyl chains $(n=16,18)$ were much less. Of these two cationic surfactants, the enhancement effects of dimethyldistearylammonium (DMDSA) were slightly greater than those of DMDPA.

Effects of Dimethyldialkylammoniums on Erythrocyte Hemolysis Interaction of dimethyldialkylammoniums with

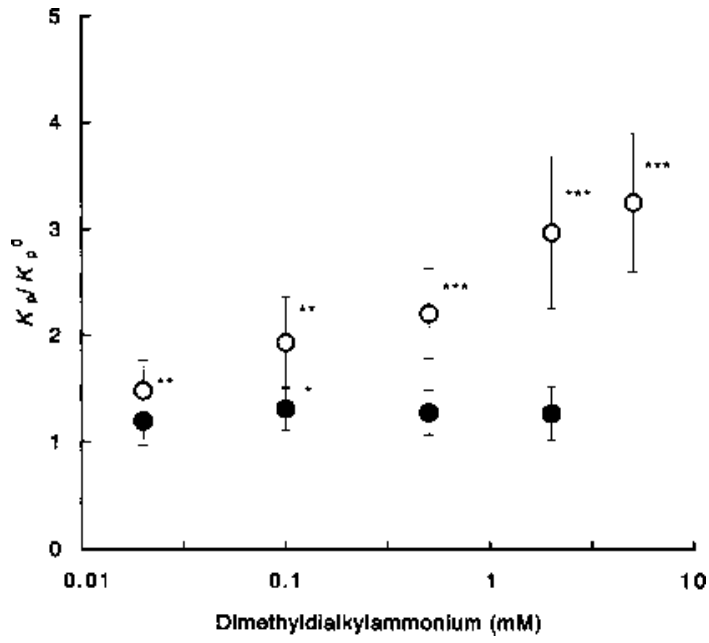

Fig. 2. Dose-Dependent Effects of DMDDA ( $\bigcirc)$ and DMDPA ( ) on the Apparent Permeability Coefficient $\left(K_{\mathrm{p}}\right)$ of Benzoic Acid

Data, which represent the ratio to the apparent permeability coefficient in the absence of dimethyldialkylammoniums $\left(K_{\mathrm{p}}^{0}\right)$, are means \pm S.D. of four experiments. The significance of the difference between the permeability coefficient of benzoic acid without the surfactant and that with the surfactant was determined by Student's $t$-test. $* p<0.05$, $* * p<0.01, * * * p<0.001$.

Table 1. Solubility $\left(C_{\mathrm{d}}\right)$, Flux $(J)$, and Apparent Permeability Coefficients $\left(K_{\mathrm{p}}\right)$ of Benzoic Acid in the Presence or Absence of Dimethyldialkylammoniums $\left(\mathrm{CH}_{3}\right)_{2} \mathrm{~N}^{+}\left(\mathrm{C}_{n} \mathrm{H}_{2 n+1}\right)_{2} 2 \mathrm{~mm}$

\begin{tabular}{|c|c|c|c|c|}
\hline Surfactant & $n^{a)}$ & $C_{\mathrm{d}}\left(\mu \mathrm{mol} \cdot \mathrm{ml}^{-1}\right)$ & $J\left(\mu \mathrm{mol} \cdot \mathrm{cm}^{-2} \mathrm{~h}^{-1}\right)$ & $K_{\mathrm{p}}\left(\mathrm{cm} \cdot \mathrm{h}^{-1}\right)$ \\
\hline None & - & $34.7 \pm 1.6$ & $3.7 \pm 0.7$ & $0.106 \pm 0.020$ \\
\hline DMDDA & 10 & $38.0 \pm 1.5$ & $12.0 \pm 1.8 * * *$ & $0.315 \pm 0.048 * * *$ \\
\hline DMDLA & 12 & $41.7 \pm 0.6^{* *}$ & $10.6 \pm 1.3 * * *$ & $0.254 \pm 0.030 * * *$ \\
\hline DMDMA & 14 & $40.3 \pm 0.5 * *$ & $10.5 \pm 0.8^{* * *}$ & $0.261 \pm 0.021 * * *$ \\
\hline DMDPA & 16 & $38.9 \pm 0.1^{*}$ & $5.2 \pm 0.6^{*}$ & $0.135 \pm 0.014$ \\
\hline DMDSA & 18 & $39.6 \pm 0.3^{*}$ & $6.1 \pm 0.4^{* *}$ & $0.154 \pm 0.011 *$ \\
\hline $\mathrm{CTMA}^{b)}$ & - & $38.1 \pm 2.1$ & $11.2 \pm 1.5^{* * *}$ & $0.293 \pm 0.036^{* * *}$ \\
\hline
\end{tabular}

Data are means \pm S.D. of four experiments. Statistical significances of differences of values from control values were determined by Student's $t$-test: $* p<0.05, * * p<0.01$, $* * * p<0.001$. a) Number of carbon atoms in each alkyl chain. b) Data for cetyltrimethylammonium (CTMA) were cited from ref. 2. 
Table 2. Hemolysis Induced by Dimethyldialkylammoniums $\left(\mathrm{CH}_{3}\right)_{2} \mathrm{~N}^{+}-$ $\left(\mathrm{C}_{n} \mathrm{H}_{2 n+1}\right)_{2} 2 \mathrm{~mm}$ after Incubation of Guinea Pig Erythrocytes for $1 \mathrm{~min}$ or $60 \mathrm{~min}$ at $37^{\circ} \mathrm{C}$

\begin{tabular}{llrr}
\hline \hline Surfactant & $n^{a)}$ & \multicolumn{2}{c}{$\%$ Hemolysis } \\
& & After 1 min & After 60 min \\
\hline DMDDA & 10 & $92.6 \pm 4.2$ & $100.0 \pm 5.7$ \\
DMDLA & 12 & $101.1 \pm 2.7$ & $99.8 \pm 0.3$ \\
DMDMA & 14 & $101.8 \pm 3.2$ & $99.3 \pm 2.1$ \\
DMDPA & 16 & $3.1 \pm 0.6$ & $99.0 \pm 2.0$ \\
DMDSA & 18 & $33.1 \pm 21.7$ & $96.5 \pm 4.5$ \\
\hline
\end{tabular}

Data are means \pm S.D. of four experiments. $a$ ) Number of carbon atoms in each alkyl chain.

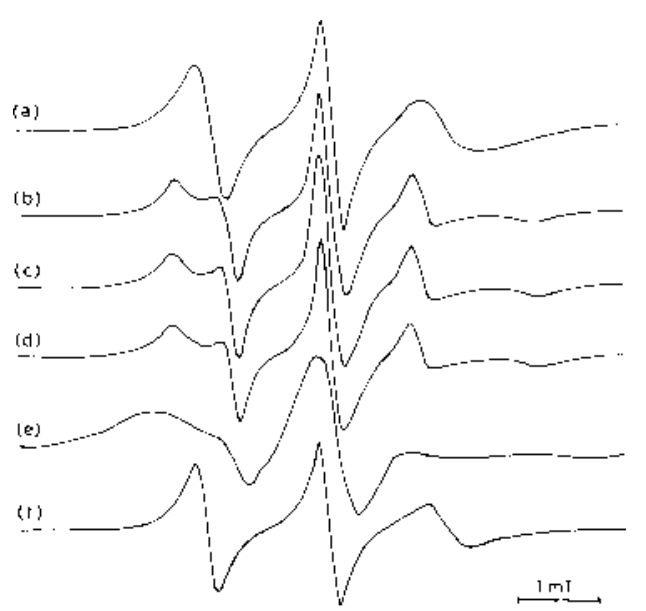

Fig. 3. ESR Spectra of 5-NS in DMDDA (a), DMDLA (b), DMDMA (c), DMDPA (d), DMDSA (e), and CTMA (f)

All at a concentration of $2 \mathrm{~mm}$.

the lipid lamella in the stratum corneum appears to be involved in their permeation enhancement process. Since erythrocytes have been used as a good model system to reveal the interaction of amphiphiles with the lipid bilayer of various biological membranes, ${ }^{14)}$ including the lipid lamella of the stratum corneum, ${ }^{8)}$ we next examined the hemolytic effects of the double-chained cationic surfactants on guinea pig erythrocytes. As shown in Table 2, hemolysis was induced immediately after the addition of dimethyldialkylammoniums with relatively shorter alkyl chains $(n=10-14)$. On the other hand, hemolysis was induced gradually with the addition of the long-chain cationic surfactants $(n=16,18)$. Marked aggregation of erythrocytes was induced by these long-chain surfactants immediately after their addition. The hemolytic activity of DMDSA, which stimulated skin permeation slightly more than that of DMDPA, was slightly greater than DMDPA. These findings indicate that the hemolytic activity of dimethyldialkylammoniums corresponds to their enhancement effects on drug skin permeation.

ESR Spectra of 5-NS in Dimethyldialkylammoniums To determine the characteristics of the molecular assemblies of dimethyldialkylammoniums, we observed the ESR spectra of 5-NS in the sonicated surfactants. 5-NS is known to have relatively isotropic ESR spectra in surfactant micelles, whereas it has more anisotropic spectra in bilayer vesicles. ${ }^{15}$ ) The ESR spectra of 5-NS in dimethyldialkylammoniums $2 \mathrm{~mm}$ are shown in Fig. 3 together with that in the singlechained cationic surfactant CTMA $2 \mathrm{~mm}$, which forms mi- celles above its critical micelle concentration $(0.04 \mathrm{~mm}) .{ }^{2)}$ As shown in Fig. 3a, the spectrum of DMDDA $(n=10)$, which resembled that in CTMA (Fig. 3f), was much more isotropic than those of the other dimethyldialkylammoniums. This suggests that DMDDA, which has relatively short alkyl chains, forms micelles at $2 \mathrm{mM}$. Except for DMDSA $(n=18)$, the other three dimethyldialkylammoniums, which have longer alkyl chains, appear to form vesicles based on their anisotropic ESR signals (Figs. $3 \mathrm{~b}-\mathrm{d}$ ). The mean values ( \pm S.D.) of the order parameters of 5-NS in the vesicles of these dimethyldialkylammoniums in four experiments were $0.416 \pm 0.002$ for DMDLA, $0.446 \pm 0.003$ for DMDMA, and $0.464 \pm 0.002$ for DMDPA. These values were much smaller than that in egg yolk phosphatidylcholine $(0.578 \pm 0.004)$. Therefore the molecular packing of the bilayer of these vesicles appears to be loose due to the cationic charge repulsion. On the other hand, DMDSA, which has longest alkyl chains among the five dimethyldialkylammoniums tested, is present in gel state based on its broad ESR spectrum (Fig. 3e). This finding is consistent with the previous finding that DMDSA does not form vesicles but forms aggregate structures below $55^{\circ} \mathrm{C} .^{16)}$

\section{Discussion}

The present findings revealed marked enhancement effects of dimethyldialkylammoniums with relatively shorter alkyl chains such as DMDDA $(n=10)$ on the skin permeation of benzoic acid. On the other hand, the enhancement effects of dimethyldialkylammoniums such as DMDPA $(n=16)$ which have longer alkyl chains were much less. The absorption enhancement effects of these cationic surfactants correspond to their hemolytic effects. These surfactants appear to induce hemolysis due to penetration of the monomers into the erythrocyte membrane and interaction with membrane components, as suggested for single-chained surfactants. ${ }^{17)}$ Equilibrium is present between vesicles (or micelles) and the surfactant monomers, and more surfactant molecules appear to present as monomers in dimethyldialkylammoniums that have shorter alkyl chains. Furthermore, vesicles of surfactants with shorter alkyl chains appear unstable due to their loose molecular packing and therefore may easily become monomers. Thus their interaction with skin components is likely to occur by penetration of the monomers into the stratum corneum, like the single-chained cationic surfactants. As suggested for the single-chained surfactants, monomers of dimethyldialkylammoniums with relatively shorter alkyl chains may interact with proteins of the stratum corneum as well as with the lipid lamella, which will stimulate the permeation of relatively hydrophilic drugs like benzoic acid used in this study. $1,2,18$ )

On the other hand, dimethyldialkylammoniums with longer alkyl chains such as DMDPA appear to form more stable vesicles or aggregate structures. As suggested by the aggregation of erythrocytes by these surfactants and their relatively weak hemolytic activity, penetration of the surfactant molecules into the stratum corneum and interaction with skin components may be much less compared with dimethyldialkylammoniums with shorter alkyl chains. The formation of relatively stable vesicles by DMDPA may be the reason for its lesser permeation enhancement effect compared with DMDSA. 
Based on the present results, it is suggested that dimethyldialkylammoniums stimulate transdermal drug permeation not as cationic vesicles but as surfactant monomers, at least when drugs are present mostly as undissociated molecules in the donor compartments as shown in this study. Since toxic effects such as hemolytic effects and irritation are basic problems, the development of skin permeation enhancement systems working as stable cationic vesicles may be important for the clinical application of these double-chained cationic surfactants.

Acknowledgments This work was supported in part by a grant from the Ministry of Education, Science, Culture and Sport of Japan (10672030) and the Promotion and Mutual Aid Corporation for Private Schools.

\section{References}

1) Ashton P., Walters K. A., Brain K. R., Hadgraft J., Int. J. Pharmaceut., 87, 261-264 (1992).

2) Kitagawa S., Kasamaki M., Ikarashi A., Chem. Pharm. Bull., 48, 1698-1701 (2000)

3) Polefka T. G., Surfactant Sci. Ser., 82, 433-468 (1999).

4) Hong K., Zheng W., Baker A., Papahadjopoulos D., FEBS Lett., 400 , 233-237 (1997).

5) Katsel P. L., Greenstein R. J., Biotechnol. Annu. Rev., 5, 197-220
(2000).

6) Wertz P. W., Swartzendruber D. C., Madison K. C., Downing D. T., J. Invest. Dermatol., 89, 419-425 (1987).

7) Rehfeld S. J., Plachy W. Z., Williams M. L., Elias P. M., J. Invest. Dermatol., 91, 499-505 (1988).

8) Tokunaga H., Chung Y., Uchino T., Ando M., J. Jpn. Cos. Sci. Soc., 24, 14-20 (2000).

9) Kitagawa S., Hosokai A., Kaseda Y., Yamamoto N., Kaneko Y., Matsuoka, E., Int. J. Pharmaceut., 161, 115-122 (1998).

10) Vandenbelt J. M., Henrich C., Vandenberg S. G., Anal. Chem., 25, 726-727 (1954).

11) Kitagawa S., Yokochi N., Murooka N., Int. J. Pharmaceut., 126, 4956 (1995).

12) Kitagawa S., Kametani F., Tsuchiya K., Sakurai H., Biochim. Biophys. Acta, 1027, 123-129 (1990).

13) Crosby W. H., Munn J. I., Furth F. W., U. S. Armed Forces Med. J., 5, 693-703 (1954).

14) Isomaa B., Hägerstrand H., Mäkelä J.-H., Biophys. Membr. Trans., 11, 273-289 (1992).

15) Ueno M., Kashiwagi H., Nihon Yukagakkaishi, 49, 1131-1139 (2000).

16) Andersson M., Hammarström L., Edwards K., J. Phys. Chem., 99, 14531-14538 (1995).

17) Isomaa B., Hägerstrand H., Paatero G., Biochim. Biophys. Acta, 899, 93-103 (1987).

18) Kitagawa S., Ikarashi A., Chem. Pharm. Bull., 49, 165-168 (2001). 